
\title{
25 Research Soure \\ Integrated analysis of ceRNA network in lung adenocarcinoma based on bioinformatics analysis
}

Huixin Wang

Shenyang Medical College

Qian Li

Shenyang Medical College

Xiaowen Hou

Shenyang Medical College

Xinzhu Shi

Shenyang Medical College

Xu Feng ( $\triangle$ fxsymc@163.com )

Shenyang Medical College https://orcid.org/0000-0001-5139-4191

\section{Research Article}

Keywords: bioinformatics, lung adenocarcinoma, competitive endogenous RNA, long non-coding RNA, AFAP1-AS1

Posted Date: December 8th, 2021

DOI: https://doi.org/10.21203/rs.3.rs-1069321/v1

License: (c) (1) This work is licensed under a Creative Commons Attribution 4.0 International License.

Read Full License 


\section{Abstract \\ Background}

Increasing studies have revealed that long noncoding RNA (IncRNAs) can bind to microRNA (miRNAs) as competitive endogenous RNA (ceRNA), which can affect the expression of mRNAs. These IncRNA related ceRNAs theoretically play an important role in the occurrence and progress of cancer. However, the roles and functions of ceRNA network in lung adenocarcinoma (LUAD) are not completely clear.

\section{Methods}

1. The chip data were downloaded from the Gene Expression Omnibus (GEO). Different IncRNAs and mRNAs were analyzed by GEO2R, and then the target miRNAs and target mRNAs were predicted. The ceRNA network was constructed and subjected to enrichment analysis. 2. The IncRNA AFAP1-AS1 gene Knockout was constructed. The siRNA was constructed and transfected into LUAD cell A549 by cell transfection. The CCK8, Transwell, scratch assay and flow cytometry were used to gauging the ability of cell proliferation, invasion, migration, and apoptosis.

\section{Results}

In total, 6 IncRNAs and 494 mRNAs were identified as differentially expressed RNAs (DERNAs) in LUAD. A total of 6 DElncRNAs interacted with 22 DEmiRNAs and 22 DEmiRNAs interacted with 55 DEmRNAs were involved in the ceRNA network. These DEmRNAs were found to be related to cancer-related pathways, such as signaling pathways that regulate stem cell pluripotency, TGF- $\beta$ signaling pathways, and protein digestion and absorption. Knockout of AFAP1-AS1 inhibits cell proliferation, invasion and migration. AFAP1-AS1 promotes apoptosis.

\section{Conclusions}

In this study, we constructed a IncRNA-miRNA-mRNA ceRNA network which may help further research on the mechanism of LUAD. In addition, we identified AFAP1-AS1 as an oncogenic IncRNA in LUAD.

\section{Introduction}

Lung cancer has highly incidence rate and mortality rate in the world [1]. Non-small cell lung carcinoma (NSCLC) account for $85 \%$ of lung cancer cases [2]. Lung adenocarcinoma (LUAD) is the main histological subtype of NSCLC [2]. Despite the development in diagnosis and treatment in recent years, the prognosis of LUAD patients is still poor. Statistics has shown that the 5-year survival rate is less than $20 \%$ in LUAD [3]. Insufficient understanding of the biological mechanism of LUAD has limited the improvement of therapeutic efficacy. 
LncRNAs are a class of single-stranded RNA molecules that have no protein-coding capacity. In the last few days, investigators discovered that LncRNAs can participate in many important biological processes, such as epigenetic regulation, transcriptional regulation, and post-transcriptional regulation, etc. [4]. Further studies have found that IncRNAs promote or inhibit the development of LUAD by participating in a variety of cytological processes including chromatin modification, gene expression regulation, transcriptional activation, transcriptional interference, intranuclear trafficking, and cell differentiation, affecting cancer metastasis and invasion, proliferation and apoptosis of cancer cells, cancer angiogenesis, and regulating cancer drug resistance, providing a new strategy for LUAD treatment [7-9]. Numerous studies have shown that IncRNAs can adsorb multiple miRNAs, and each miRNA has multiple downstream target genes combined, forming a quite complex regulatory network of IncRNAs that become ceRNAs, which affect and regulate the expression of mRNAs and target genes. These ceRNAs associated with IncRNAs are theoretically closely related to the development and progression of cancer [10]. Therefore, IncRNAs acting as ceRNAs have diverse biological functions that deserve further exploration.

Herein, this study predicted differentially expressed genes of IncRNAs and mRNAs in LUAD patients through the GEO database. Then we constructed a ceRNA network. The most differentially expressed genes were verified by experiments. These IncRNAs involved in the ceRNA network may become potential therapeutic targets or diagnostic biomarkers for LUAD.

\section{Materials And Methods}

\subsection{Datasets Selection}

Gene expression microarray datasets were downloaded from the GEO database. The inclusion criterias were including: (1) the studies were about lung adenocarcinoma; (2) tissue samples including cancer and corresponding adjacent or normal tissues; (3) the total number of samples was not less than 40; (4) the dataset must include IncRNAs and mRNAs.

\subsection{Analysis of Differential Gene Expression in LUAD}

Differentially expressed IncRNAs (DElncRNAs) and DEmRNAs were obtained by gene expression profiling of the GEO2R analysis datasets with screening conditions of $|\operatorname{LogFC}|>1.5$ and adjusted $P$-value $<0.05$.

\subsection{Constructing ceRNA networks}

The IncRNA-miRNA-mRNA ceRNA network was based on theory that IncRNAs can directly interact by invoking miRNAs sponges to regulate mRNA activity. (1) miRcode (http://www.mircode.org/) was applied to predict target miRNAs of the DElncRNAs. (2) miRDB (http://mirdb.org/), miTarBase (http://www.mirtarbase.org/), and Targetscan (http://www.targetscan.org/) were applied to predict target mRNAs of miRNAs. The intersections of the three databases were taken. (3) LncRNAs, miRNAs and mRNAs selected were used to constructed the ceRNA network by using Cytoscape v3.8.2.

\subsection{Constructing PPI Networks}


Protein-protein interaction (PPI) analysis is an important tool for discovering LUAD-related differentially expressed genes of different modules based on protein networks. PPI networks were constructed to identify key coding genes and important gene modules in LUAD compared to paracancerous tissues. We used the String database and visualized using Cytoscape v3.8.2 to construct PPI networks.

\subsection{Functional Enrichment Analysis}

Functional enrichment analysis, including KEGG pathway and GO(including molecular functions, MF; cellular components, $\mathrm{CC}$ and biological processes, BP), were performed for the IncRNAs and mRNAs from the regulatory network with DAVID (Database for Annotation, Visualization and Integration Discovery, https://david.ncifcrf.gov/). $P$-value $<0.05$ was set as the threshold.

\subsection{Cell culture and transfection}

A549 cells and MRC- 5 cells were provided by Procell. They were cultured in RPMI-1640 (Sigma-Aldrich, Whitehouse Station, New Jersey) medium containing $10 \%$ fetal bovine serum (FBS, Gibco, Carlsbad, California). Cells in the logarithmic growth phase were subjected to transfection at a confluency of 70$80 \%$. Transfection vectors and Lipofectamine 6000 (Beyotime Biotechnology, China) were respectively diluted in Opti-MEM. After mixture and incubation for $20 \mathrm{~min}$, the cells were plated in each well.

\subsection{Reverse transcription-quantitative polymerase chain reaction (RT-qPCR).}

Total RNA was extracted from NSCLC A549 cell and lung cells of human MRC-5 using trizol reagent, and was quantified using NanoDrop 2000 spectropho tometer. Total RNA was reverse transcribed into complementary deoxyribonucleic acid (cDNA) using PrimeScript RT reagent. The obtained cDNA was further amplified by quantitative PCR using SYBR Premix Ex Taq. The relative levels were quantitatively analyzed using the 2- $\Delta \Delta \mathrm{Ct}$ method. Glyceraldehyde 3-phosphate dehydrogenase (GAPDH) was used as an internal reference.

Primer sequences:

GAPDH

Follows: CAGGAGGCATTGCTGATGAT

Reverse: GAAGGCTGGGGCTCATTT

AFAP1-AS1

Follows: CTCGGGGTTACCAGCAATGT

Reverse: AGTTGGCTGATTCAACAGGCT

\subsection{Cell proliferation assay}


Cells were seeded in a 96-well plate with $2.0 \times 10^{3}$ cells/well and incubated with 0, 0.5, 1, 1.5, 2 and 2.5 $\mathrm{mg} / \mathrm{ml}$, respectively. Each experiment was performed in triplicate. Absorbance at 450nm was recorded using CCK-8 kit , the viability curve was plotted. Cell viability was measured at 1 day, 2 days, 3 days, 4 days and 5 days.

\subsection{Cell migration assay}

Cells were seeded in a 6 -well plate with $5.0 \times 10^{4}$ cells/well and incubated. Mark drew a straight line in the 6-well plate. The dropped cells were washed with PBS. The scratch width of cells was observed at the time of $0 h$ and $48 h(n=5)$.

\subsection{Cell invasion assay}

For the invasion assay, $4.0 \times 10^{4}$ cells were added to the top of the Transwell membrane in the upper chamber coated with Matrigel. A complete medium was added to the bottom wells as stimulation of invasion. The cells were then incubated at $37^{\circ} \mathrm{C}$ for $48 \mathrm{~h}$. The cells on the upper surface were washed away, while the cells on the bottom surface were fixed with $20 \%$ methanol (5 minutes) and stained with $0.1 \%$ crystal violet. Then stained cells were observed and counted under an inverted microscope, and five different microscopic views were randomly selected for analysis, including different multiples, and experiments were performed in the triplicate assay $(n=3)$.

\subsection{Cell apoptosis assay}

Apoptosis was analyzed by flow cytometry with Annexin V-PI apoptosis detection kit. Cells were treated with siRNA for 48h. Cells were then collected and stained with AnnexinV-PI according to the instructions. The early and late apoptotic cells were detected by flow cytometry after double staining.

\subsection{Statistical analysis}

Student's t-test and one-way ANOVA analysis were performed to analyze the data using SPSS 22.0 software. $P<0.05$ was considered statistically significant.

\section{Results}

\subsection{Differentially expressed genes in LUAD}

Gene expression microarray datasets GSE27262 (including 25 normal tissues and 25 lung adenocarcinoma tissues; GPL: 570) and GSE19804 (including 60 normal tissues and 60 lung adenocarcinoma tissues; GPL: 570) were downloaded from the GEO database (https://www.ncbi.nlm.nih.gov/geo/).

There were 1051 DEmRNAs (358 up-regulated and 693 down-regulated mRNAs) and 19 DElncRNAs (9 upregulated and 10 down-regulated IncRNAs) in the GSE27262 dataset, and 510 DEmRNAs (129 up- 
regulated and 381 down-regulated mRNAs) and 6 DElncRNAs (3 up-regulated and 3 down-regulated IncRNAs) in the GSE19804 dataset.

Figure 1

The Venn diagram showed the intersection of DEmRNAs and DElncRNAs, with a total of 494 DEmRNAs and 6 DElncRNAs. (Figure 2).

Figure 2

\subsection{Construction of ceRNA network}

A total of 6 DElncRNAs interacted with 22 DEmiRNAs (Table 1) and 22 DEmiRNAs interacted with 55 DEmRNAs (Table 2) were involved in the ceRNA network, which contains 83 nodes and 155 edges. (Figure $3)$.

Table 1

Table 2

Figure 3

\subsection{GO and KEGG pathway analysis}

GO and KEGG pathway enrichment analyses revealed that the DEmRNAs involved in ceRNA network were remarkably associated with $37 \mathrm{BPs}$, including BMP signaling pathway, positive regulation of cell migration, and negative regulation of axon extension involved in axon guidance. The enrichment of MFs is mainly related to heparin-binding, signaling protein receptor binding, chemorepellent activity, SMAD binding, growth factor activity, receptor binding. We found that the most enriched CCs were heparinbinding, semaphorin receptor binding, and chemorepellent activity. KEGG enrichment analysis showed that DEmRNAs were significantly enriched in regulating stem cell pluripotency, TGF- $\beta$ signaling pathway and protein digestion and absorption. In addition, we constructed a PPI network to identify the interaction between mRNA proteins in the ceRNA network. We found some genes with higher composite scores, including FGF2, COL1A2, BMP2, KLF4, CXCL5 and MMP11, were mainly enriched in "TGF- $\beta$ signaling pathway", as shown in Figure 6.

Figure 4

Figure 5

\subsection{Expression of IncRNA AFAP1-AS1 in LUAD cells}

The expression of IncRNA AFAP1-AS1 in A549 cells was $1.37 \pm 0.15$, which was significantly higher than that in MRC- 5 by $0.57 \pm 0.13(P<0.05)$, as shown in Figure 6 . 
Figure 6

\subsection{Transfection effect of siRNA}

The expression of IncRNA AFAP1-AS1 in siRNA-NC, siRNA-1, siRNA-2 and siRNA-3 group were $1.23 \pm 0.11$, $0.55 \pm 0.09,0.31 \pm 0.04$ and $0.44 \pm 0.02(F=90.05, P<0.001)$. Compared with siRNA-NC group, the expression of IncRNA AFAP1-AS1 decreased significantly in siRNA-2 group, as shown in Figure 6.

Figure 7

\subsection{Silencing of AFAP1-AS1 promotes tumor growth}

The results of tumor growth in LUAD cells with altered expression of AFAP1-AS1 were shown in Figure 8. On the fourth and fifth days, the growth rate of silencing AFAP1-AS1 was significantly lower than that of the control group and the difference was statistically significant.

Figure 8

\subsection{Silencing of AFAP1-AS1 inhibition of cell migration and cell invasion}

The effect of AFAP1-AS1 on the migration ability of LUAD cells was detected by cell migration assay. As shown in Figure 9, in A549 cell line, the number of cell migration in AFAP1-AS1 siRNA-2 group was significantly lower than that in the control group. The down-regulation of AFAP1-AS1 on the invasion of LUAD cells was detected by Transwell experiment. The number of cell invasion in down-regulation of AFAP1-AS1 was lower than that in the control group, as shown in Figure $10(P<0.05)$.

Figure 9

Figure 10

\subsection{Silencing of AFAP1-AS1 promote apoptosis}

The results of apoptosis in LUAD cells with down-regulation of AFAP1-AS1 were shown in Figure 11. The suppression of AFAP1-AS1 significantly increased the apoptosis of A549 and the difference was statistically significant.

Figure 11

\section{Discussion}

Statistic showed that LUAD caused about 500,000 deaths each year all over the world [7]. Up to now, the exact pathogenesis of LUAD has remained incompletely understood. Therefore elucidating the molecular mechanisms underlying LUAD is of great importance for identifying new therapeutic targets to improve the clinical outcomes of patients. Accumulating studies indicated that IncRNAs could influence the occurrence and progression of cancer through multiple pathways [11-12]. In 2011, Salmena et al. [13] 
first proposed IncRNA-miRNA-mRNA interactions which provided a basis for the in-depth exploration of the mechanisms of various diseases, particularly cancer. Increasing studies were carried out based on this theory. Sun et al. [14] found that IncRNA H19 induced miR-675-5p up-regulation, while the level of P53 was significantly down-regulated, and p53 was identified as a target gene of miR-675. In addition, IncRNA LINC00355 and LINC00466 can target CCNE1 and HOXA10 by sponging miR-195 and miR-144, respectivelyto promote the progression of LUAD [15-16].

To the best of our knowledge, there are few ceRNA regarding LUAD. Thus, we obtained microarray datasets from the GEO database using bioinformatics analysis to mine LUAD-related IncRNAs and mRNAs with differential expression. By constructing the IncRNA-miRNA-mRNA ceRNA network, a total of 6 IncRNAs, 22 miRNAs and 55 mRNAs were identified, which included 83 nodes and 155 edges. The results of GO enrichment analysis indicated that RNAs associated with ceRNAs are mainly involved in cancer-related biological processes, for example, positive regulation of DNA transcription, signaling protein receptor binding and extracellular space. In addition, DEmRNAs involved in the ceRNA network were significantly abundant in three KEGG pathways (signaling pathway regulating stem cell pluripotency, TGF- $\beta$ signaling pathway, protein digestion and absorption). Enrichment analysis suggests that by regulating these biological processes and pathways, LUAD-specific ceRNA networks may be involved in cancer processes. Which suggested that the IncRNA-miRNA-mRNA ceRNA network may regulate the biological processes and pathways of LUAD.

LINC01207 was found to be a novel LUAD-specific IncRNA that was significantly up-regulated in LUAD, and the expression level of LINC01207 correlated with TNM stage. Both in vivo and in vitro experiments have shown that LINC01207 can promote LUAD cell proliferation [17]. Acha-Sagredo et al. [18] found that LINC00968 was down-regulated in LUAD and could inhibit the progression of LUAD through the miR-21$5 p / S M A D 7$ signaling axis. Unfortunately, there were few studies about the roles of PCAT19 and LINC00312 in LUAD. Therefore, further research is needed to clarify the role of these genes in LUAD.

The actin fiber-associated protein 1-antisense RNA1 (AFAP1-AS1), which is located on the antisense strand of chromosome 4 of humans, plays a vital role in mammalian development [19-20]. LncRNA AFAP1-AS1 has been described as an oncogene in various cancers, including gastric, colorectal, liver, hepatocellular and lung cancers [21]. High expression of AFAP1-AS1 was found to be an independent risk factor for poor patient prognosis in LUAD and could promote LUAD cell apoptosis by regulating the miR545-3p/hepatocarcinoma-derived growth factor axis [22]. To test the biological function of AFAP1-AS1, we designed siRNA that efficiently silenced AFAP1-AS1 expression in A549 cells. The expression level of AFAP1-AS1 in LUAD cells was significantly higher than that in normal lung cells. As predicted, the silence of AFAP1-AS1 inhibited proliferation, suppressed metastasis ability, reduced invasive ability and promoted apoptosis of LUAD cell line. These results indicated that AFAP1-AS1 might function as an oncogene and exhibit important role development and progression of lung cancer.

\section{Conclusion}


A IncRNA-miRNA-mRNA ceRNA regulatory network of LUAD was constructed by using bioinformatics methods. AFAP1-AS1 was an oncogene, which plays an important role in the occurrence and development of lung cancer.

\section{Abbreviations}

LncRNAs

long non-coding RNAs

ceRNA

Competing endogenous RNA

LUAD

lung adenocarcinoma

GEO

Gene Expression Omnibus

GO

Gene Ontology

KEGG

Kyoto Encyclopedia of Genes and Genomes

DERNAs

differentially expressed RNAs

SCLC

small cell lung carcinoma

NSCLC

non-small cell lung carcinoma

AFAP1-AS1

actin fiber-associated protein 1-antisense RNA1.

\section{Declarations}

\section{Acknowledgments}

This research was accomplished with the support of the Liaoning Provincial Natural Science Foundation. Meanwhile, this research was accomplished with the support of

\section{Ethics approval and consent to participate}

An ethics statement is not applicable because this study is based exclusively on published literature.

\section{Consent for publication}

All authors approved the final version manuscript and published in your journal. This manuscript does not contain any details, images, or videos that might lead to identification of an individual patient. 
Availability of data and materials

All data generated or analyzed during this study are included in this published article which can be further obtained from the corresponding author.

\section{Competing interests}

The authors have no conflicts of interest to disclose.

\section{Funding}

This work was supported by grants from the Liaoning Provincial Natural Science Foundation [No. 2019ZD-0324] and Science and Technology Innovation Fund Project for Postgraduates of Shenyang Medical College [No. Y20210509].

\section{Authors' contributions}

Huixin Wang searched the literature and wrote the first draft of the manuscript. Qian Li, Huixin Wang and Xiaowen Hou conducted the experiments and the data analysis. Xu Feng designed the study and revised the manuscript.

\section{References}

1. Siegel RL, Miller KD, Jemal A. Cancer statistics, 2019. CA Cancer J Clin. 2019;691:7-34.

2. Travis WD, Brambilla E, Nicholson AG, Yatabe Y, Austin JHM, Beasley MB, et al. WHO Panel. The 2015 World Health Organization classification of lung cancers: impact of genetic, clinical and radiologic advances since the 2004 classification. J Thorac Oncol. 2015;10(9):1243-1260.

3. Bridges MC, Daulagala AC, Kourtidis A. LNCcation: IncRNA localization and function. J Cell Biol. 2021 Feb 1;220(2):e202009045.

4. Hou G, Yang J, Tang J, He Y. LncRNA GAS6-AS2 promotes non-small-cell lung cancer cell proliferation via regulating miR-144-3p/MAPK6 axis [J]. Cell Cycle, 2021;20(2):179-193.

5. Huang Z, Zhuo W, Xu R, Wu Z, Xiong Y, Xu Z. The relationship between Long Noncoding RNA (IncRNA) Small Nucleolar RNA Host Gene 12 (SNHG12) expression in solid malignant cancers and prognosis of cancer patients: A systematic review and meta-analysis. Medicine (Baltimore). 2020;99(41):e22247.

6. Rajagopal T, Talluri S, Akshaya RL, Dunna NR. HOTAIR IncRNA: A novel oncogenic propellant in human cancer. Clin Chim Acta. 2020;503:1-18.

7. Kopp F, Mendell JT. Functional classification and experimental dissection of long noncoding RNAs. Cell. 2018;172:393-407.

8. Du Z, Sun T, Hacisuleyman E, Fei T, Wang X, Brown M, et al. Integrative analyses reveal a long noncoding RNA-mediated sponge regulatory network in prostate cancer. Nat Commun. 
2016;7:10982.

9. Batista PJ, Chang HY. Long noncoding RNAs: cellular address codes in development and disease. Cell. 2013;152:1298-307.

10. Seo D, Kim D, Chae Y, Kim W. The ceRNA network of IncRNAs and miRNAs in lung cancer. Genomics Inform. 2020;18(4):e36.

11. Shen $Y$, Peng $X$, Shen $C$. Identification and validation of immune-related IncRNA prognostic signature for breast cancer. Genomics. 2020 May;112(3):2640-2646.

12. Xu C, Liu M, Jia D, Tao T, Hao D. LncRNA TINCR SNPs and Expression Levels Are Associated with Bladder Cancer Susceptibility. Genet Test Mol Biomarkers. 2021 Jan;25(1):31-41.

13. Salmena L, Poliseno L, Tay Y, Kats L, Pandolfi PP. A ceRNA Hypothesis: The Rosetta Stone of a Hidden RNA Language? Cell. 2011;146 (3):353-8.

14. Su K, Wang N, Shao Q. The role of a ceRNA regulatory network based on IncRNA MALAT1 site in cancer progression [J]. Biomedicine \& Pharmacotherapy, 2021,137(13):111389.

15. Liang Y, Rong X, Luo Y, Li P, Han Q, Wei L, et al. A novel long non-coding RNA LINC00355 promotes proliferation of lung adenocarcinoma cells by down-regulating miR-195 and up-regulating the expression of CCNE1. Cell Signal. 2020;66:109462.

16. Ma TG, Hu YB, Guo YX. cancer-promoting activity of long noncoding RNA LINC00466 in lung adenocarcinoma via miR-144e Regulated HOXA10 Axis. Am J Pathol. 2019;189:2154-70.

17. Chi D, Zhang W, Jia Y, Cong D, Yu K, Hu S. LINC01207 Predicts Poor Prognosis and Suppresses Cell Growth and Metastasis via Regulating GSK-3 $\beta / \beta$-Catenin Signaling Pathway in Malignant Glioma. Med Sci Monit. 2020 Jun 13;26:e923189.

18. Acha-Sagredo A, Uko B, Pantazi P, Bediaga NG, Moschandrea C, Rainbow L, et al. Long non-coding RNA dysregulation is a frequent event in non-small cell lung carcinoma pathogenesis. $\mathrm{Br} \mathrm{J}$ Cancer. 2020;122(7):1050-1058.

19. Han M, Gu Y, Lu P, Li J, Cao H, Li X, et al. Exosome-mediated IncRNA AFAP1-AS1 promotes trastuzumab resistance through binding with AUF1 and activating ERBB2 translation. Mol Cancer. 2020;19(1):26.

20. Zhou Y, Shi X, Chen H, Mao B, Song X, Gao L, et al. Tumor Immune Microenvironment Characterization of Primary Lung Adenocarcinoma and Lymph Node Metastases [J]. Biomed Res Int, 2021;2021(7):1-14.

21. Wu W, Bhagat TD, Yang X, Song JH, Cheng Y, Agarwal R, et al. Hypomethylation of noncoding DNA regions and overexpression of the long noncoding RNA, AFAP1-AS1, in Barrett's esophagus and esophageal adenocarcinoma. Gastroenterology. 2013 May;144(5):956-966.e4.

22. Sun J, Min H, Yu L, Yu G, Shi Y, Sun J. The knockdown of IncRNA AFAP1-AS1 suppressed cell proliferation, migration, and invasion, and promoted apoptosis by regulating miR-545-3p/hepatomaderived growth factor axis in lung cancer [J]. Anti-Cancer Drugs, 2021;32(1):11-21. 


\section{Tables}

Table 1 Targeted miRNAs of lncRNAs in ceRNA network

\begin{tabular}{lllll}
\hline LncRNAs & miRNAs & & & \\
\hline LINC00968 hsa-miR-9-5p & hsa-miR-141-3p & hsa-miR-3619-5p & hsa-miR-4533 & hsa-miR-5011-5p \\
\hline AFAP1-AS1 hsa-miR-4695-5p hsa-miR-877-3p & hsa-miR-9500 & hsa-miR-3612 & \\
\hline LINC00312 hsa-miR-9-5p & hsa-miR-135-5P & hsa-miR-590-5p & \\
\hline LINC00511 hsa-miR-192-5p & hsa-miR-23b-3p & hsa-miR-27a-3p & hsa-miR-126-5p & \\
\hline PCAT19 hsa-miR-96-5p & hsa-miR-1299 & hsa-miR-6782-5p & hsa-miR-6736-3p & \\
\hline
\end{tabular}

Table 2 Targeted mRNAs of miRNAs in ceRNA network

\begin{tabular}{|c|c|c|c|c|c|c|c|}
\hline$\overline{\text { miRNAs }}$ & mRNAs & & & & & & \\
\hline hsa-miR-141-3p & QKI & & & & & & \\
\hline hsa-miR-3619-5p & KCNK5 & & & & & & \\
\hline hsa-miR-4533 & RTKN2 & COL1A2 & & & & & \\
\hline \multirow[t]{2}{*}{ hsa-miR-5011-5p } & SERTM1 & ADH1B & SMAD9 & GIMAP7 & NEDD4L & LRRC31 & KLF6 \\
\hline & HHIP & ID4 & SGPP2 & BMP2 & CXCL5 & KLRD1 & \\
\hline hsa-miR-4695-5p & KLRD1 & & & & & & \\
\hline hsa-miR-877-3p & S1PR1 & GOLM1 & LRRFIP1 & DLC1 & & & \\
\hline hsa-miR-9500 & CLDN11 & & & & & & \\
\hline hsa-miR-3612 & CCBE1 & SFN & & & & & \\
\hline hsa-miR-9-5p & ID4 & IGF2BP3 & & & & & \\
\hline hsa-miR-135-5P & SLC6A4 & KLF4 & MMP11 & & & & \\
\hline hsa-miR-590-5p & RECK & & & & & & \\
\hline hsa-miR-192-5p & FGF2 & B3GALNT1 & ID4 & & & & \\
\hline hsa-miR-23b-3p & SEMA6D & & & & & & \\
\hline hsa-miR-27a-3p & RNF182 & SEMA6A & TGFBR3 & SEMA6D & LIFR & ID4 & DKK2 \\
\hline hsa-miR-126-5p & PSAT1 & TMEM100 & UGT8 & & & & \\
\hline hsa-miR-96-5p & PROK2 & SLC1A1 & & & & & \\
\hline hsa-miR-1299 & MYRF & & & & & & \\
\hline hsa-miR-6782-5p & MDK & & & & & & \\
\hline hsa-miR-6736-3p & SEMA5A & CLEC14A & EMCN & SEMA6D & SOX7 & ZWINT & RGS17 \\
\hline hsa-miR-1301-3p & KLRD1 & & & & & & \\
\hline hsa-miR-6819-5p & THBS2 & BMP2 & & & & & \\
\hline hsa-miR-4659b-3p & FGF2 & DLC1 & HBEGF & PCDH17 & GREM1 & UGT8 & LGSN \\
\hline
\end{tabular}

\section{Figures}




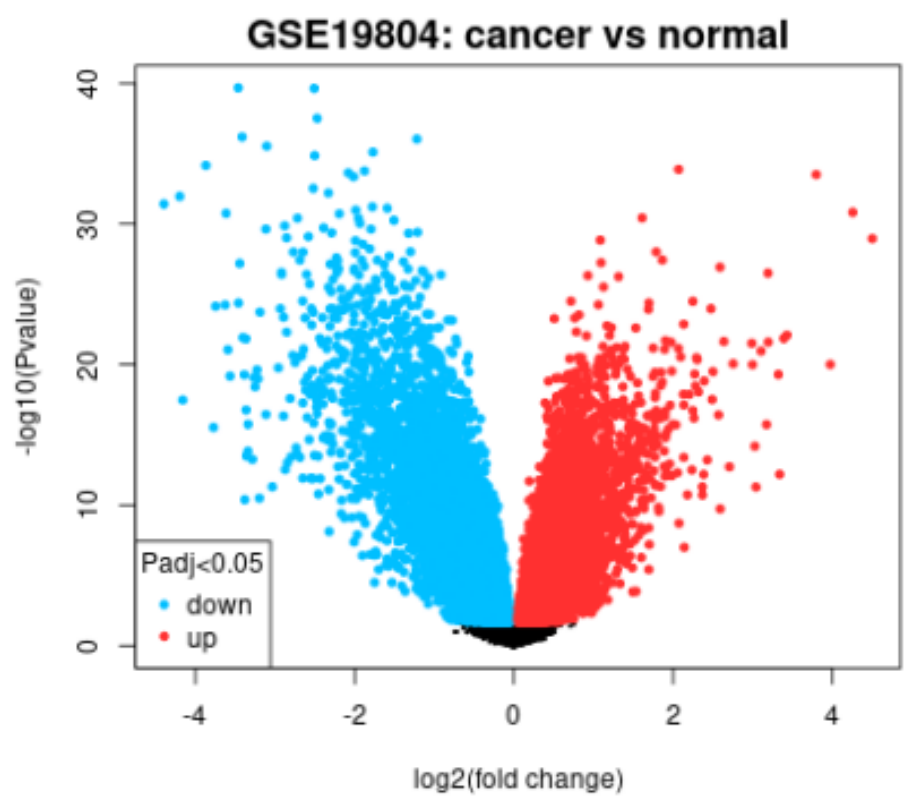

A

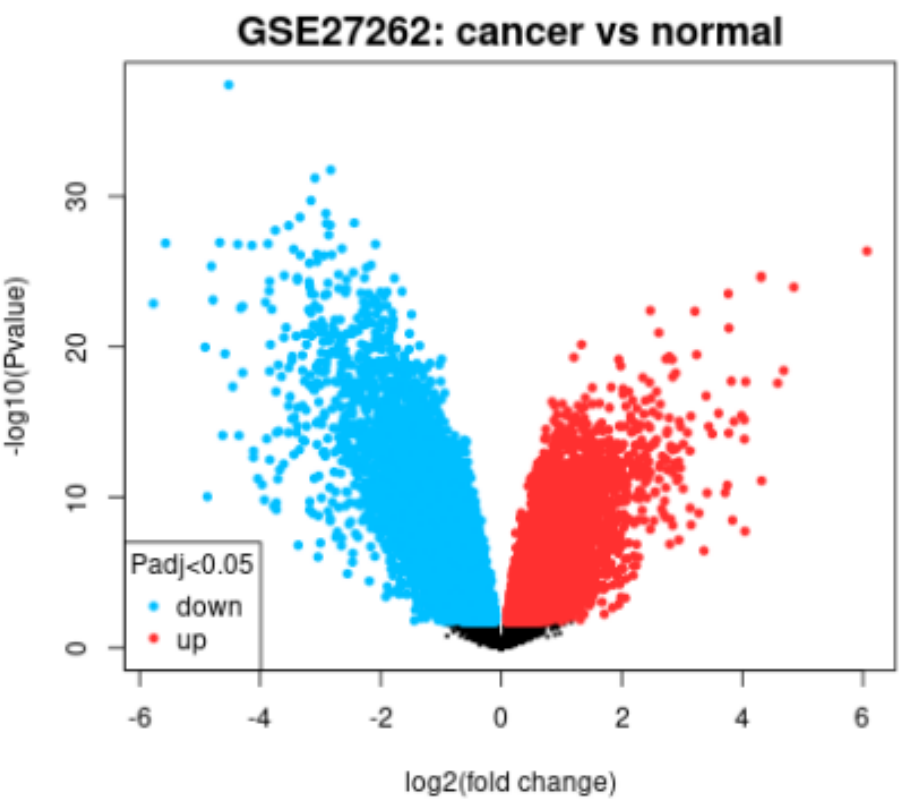

B

\section{Figure 1}

volcano plot of differentially expressed genes (A: Differentially expressed genes of GSE19804; B:

Differentially expressed genes of GSE27262; Red represents up-regulated genes and blue represents down-regulated genes. )

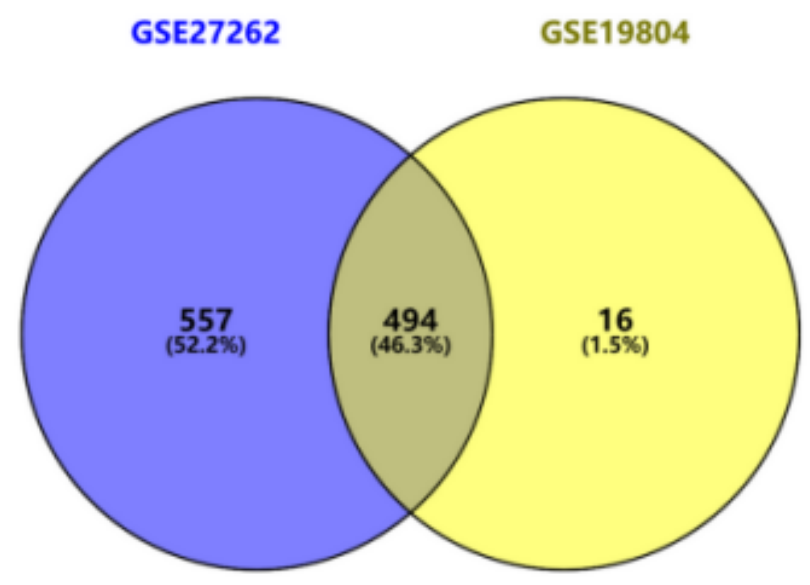

A
GSE27262

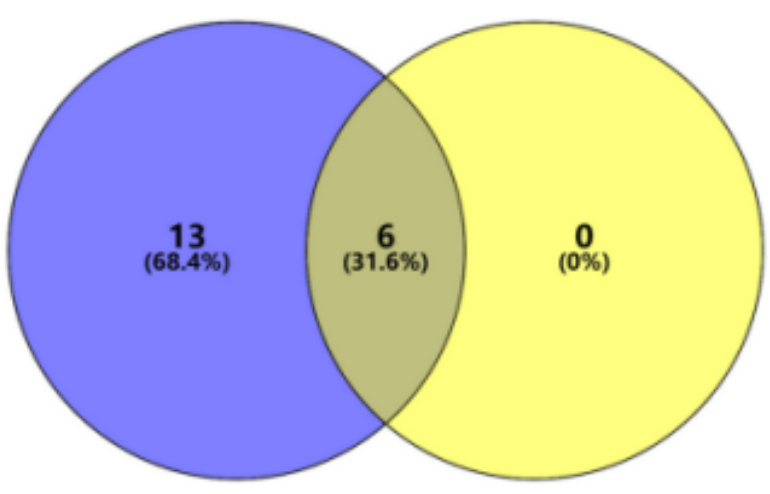

\section{Figure 2}


Venn diagram of differentially expressed genes (A: Intersection of differentially expressed mRNAs in GSE27262 and GSE19804; B: Intersection of differentially expressed IncRNAs in GSE27262 and GSE19804.)

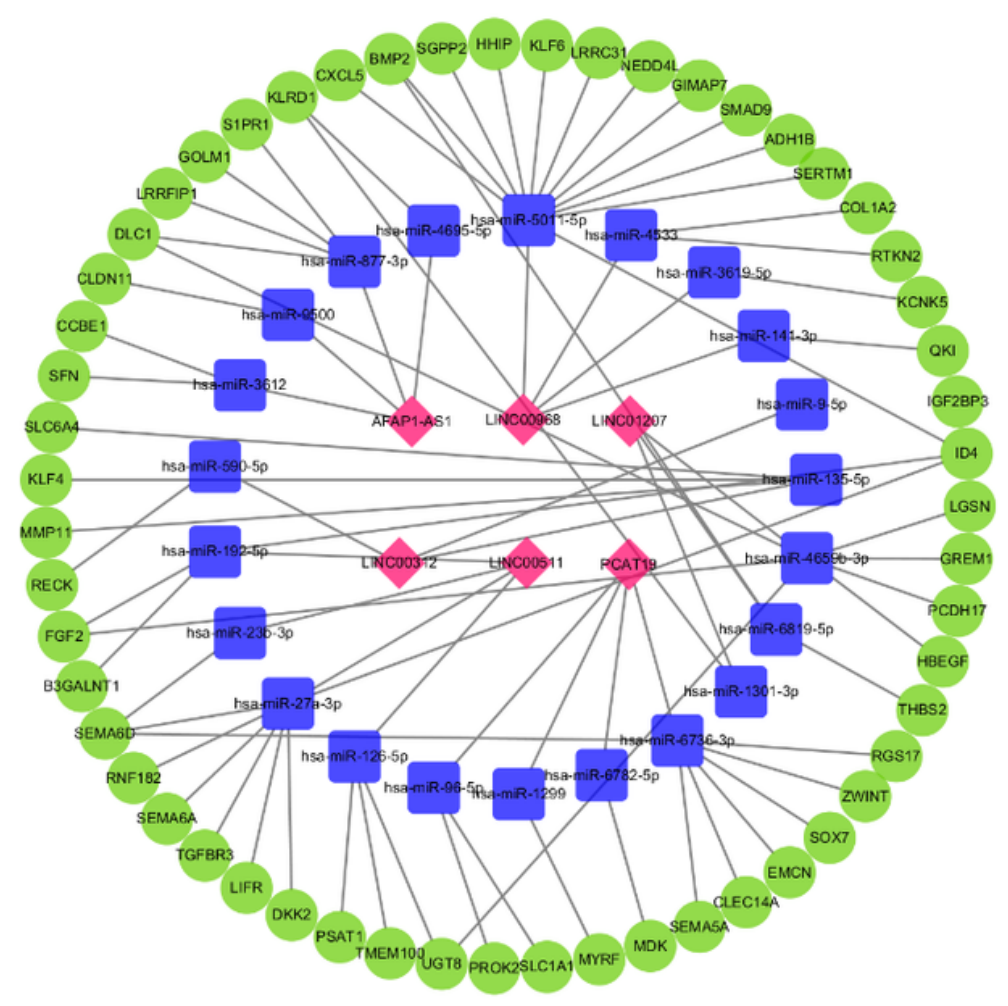

\section{Figure 3}

IncRNA-miRNA-mRNA ceRNA network constructed in LUAD. (Green nodes represent mRNAs, blue nodes represent miRNAs, and pink nodes represent IncRNAs.) 


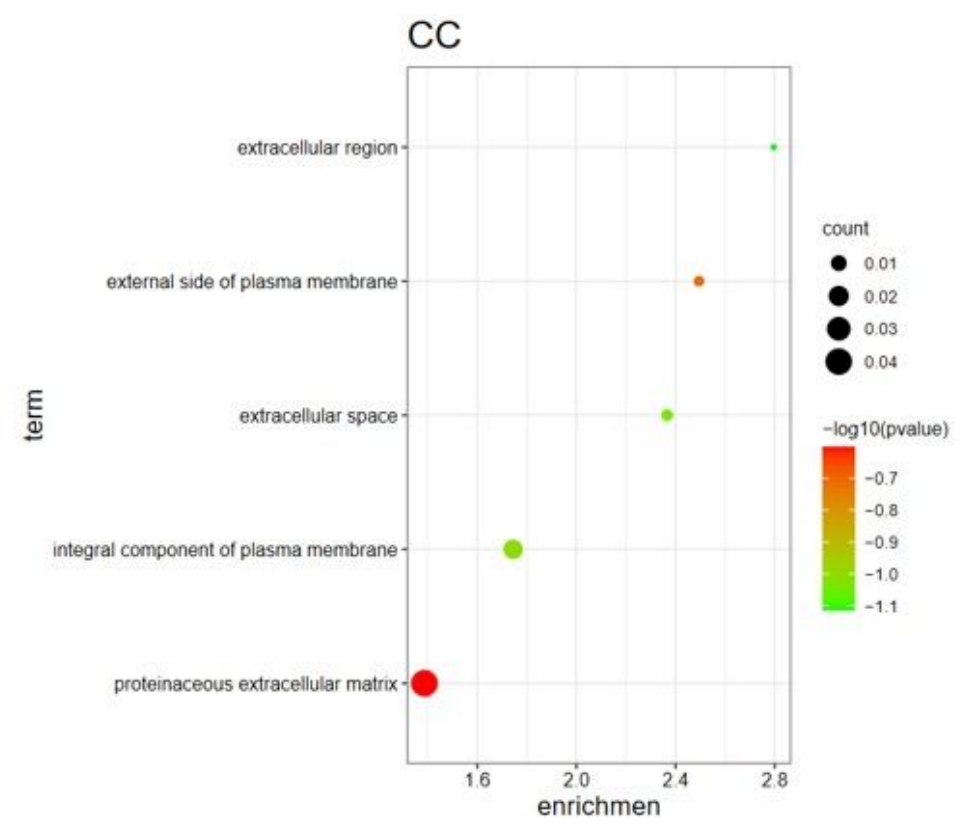

A (CC)

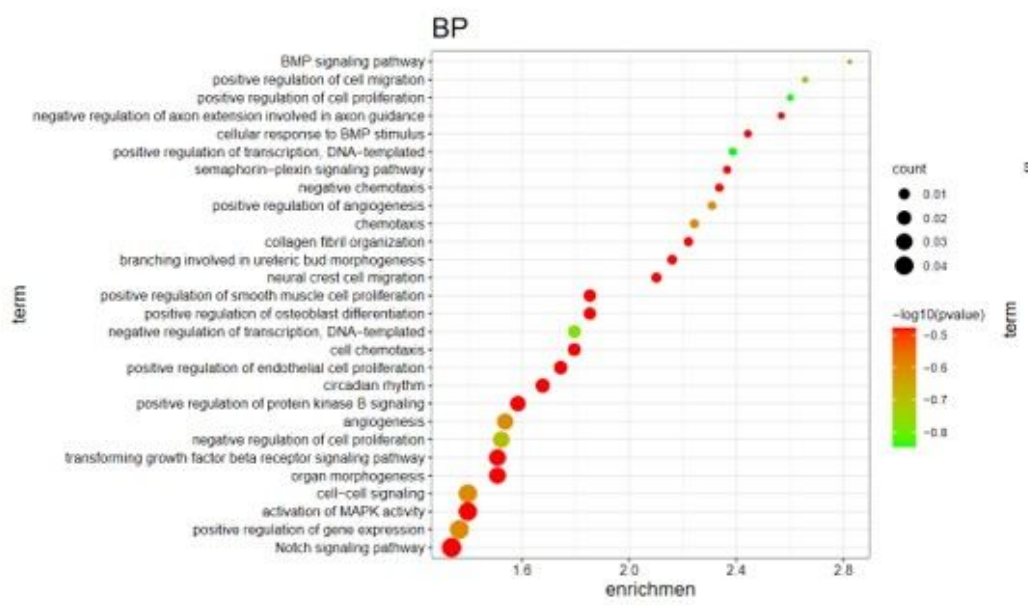

C (BP)

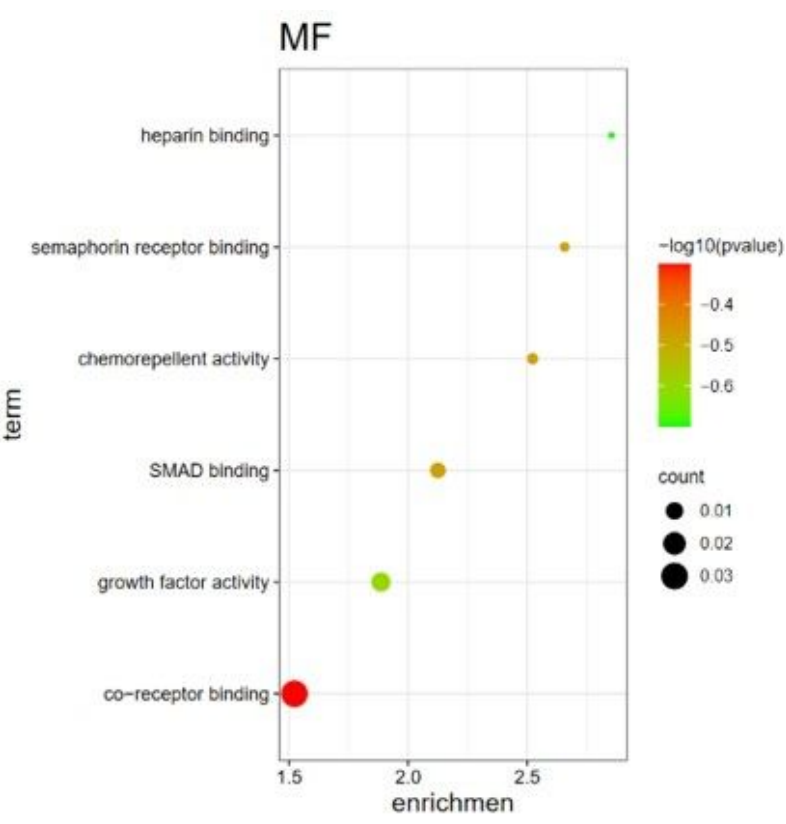

B (MF)

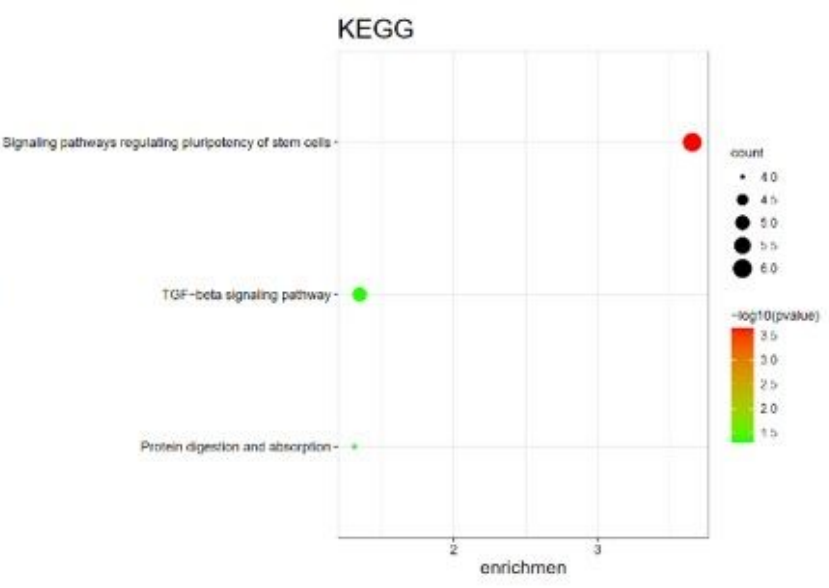

D (KEGG)

\section{Figure 4}

GO and KEGG enrichment analysis of DEmRNA in ceRNA network. ( A: Bubble plot of CC. B: Bubble plot of MF. C: Bubble plot for BP. D: Bubble diagram of KEGG. GO: Gene Ontology; BP: Biological process; CC: Cellular component; MF: Molecular function. ) 


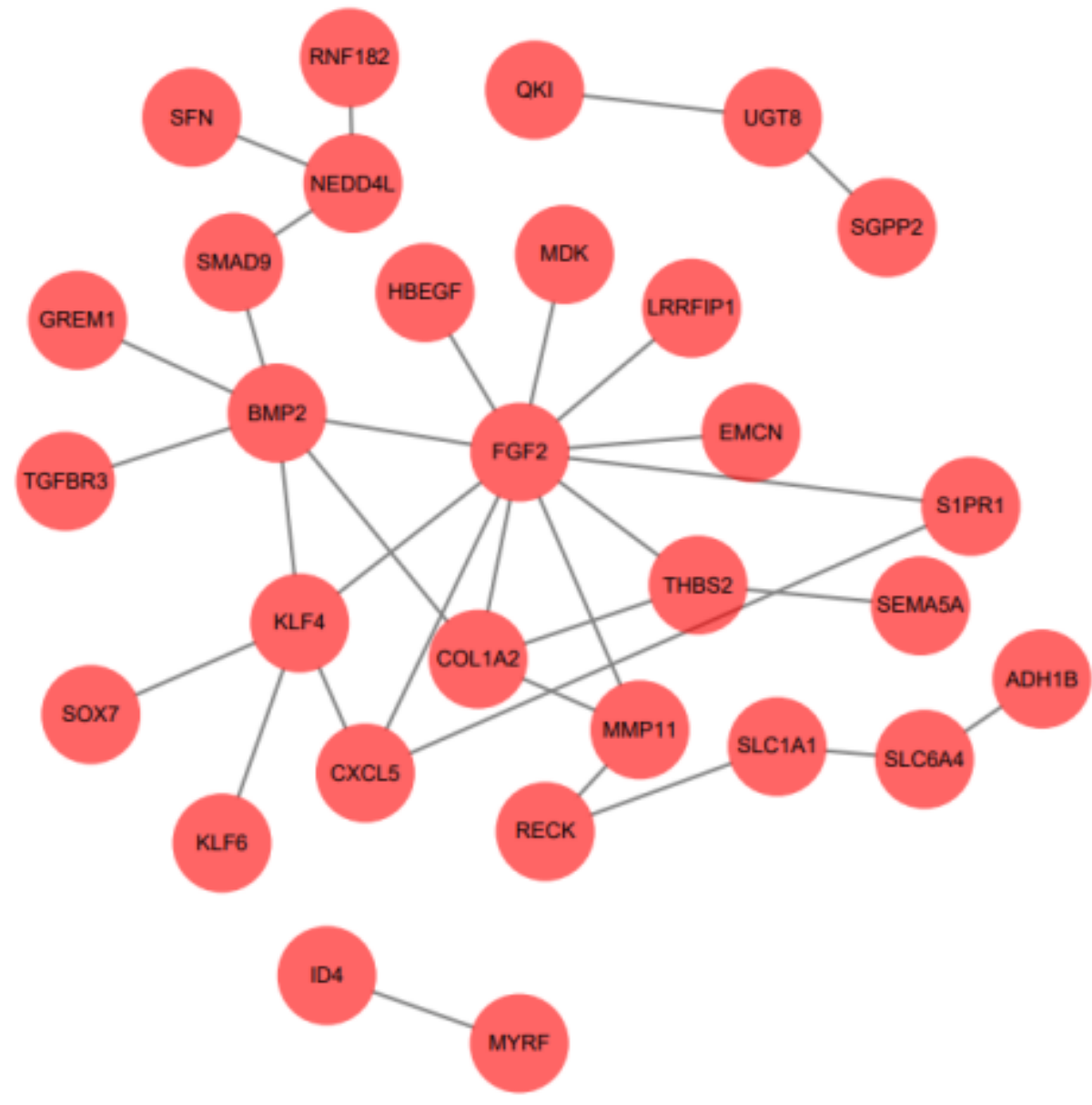

Figure 5

PPI network of mRNAs in ceRNA network. (Circles represent genes and lines represent interactions between proteins encoded by genes.) 


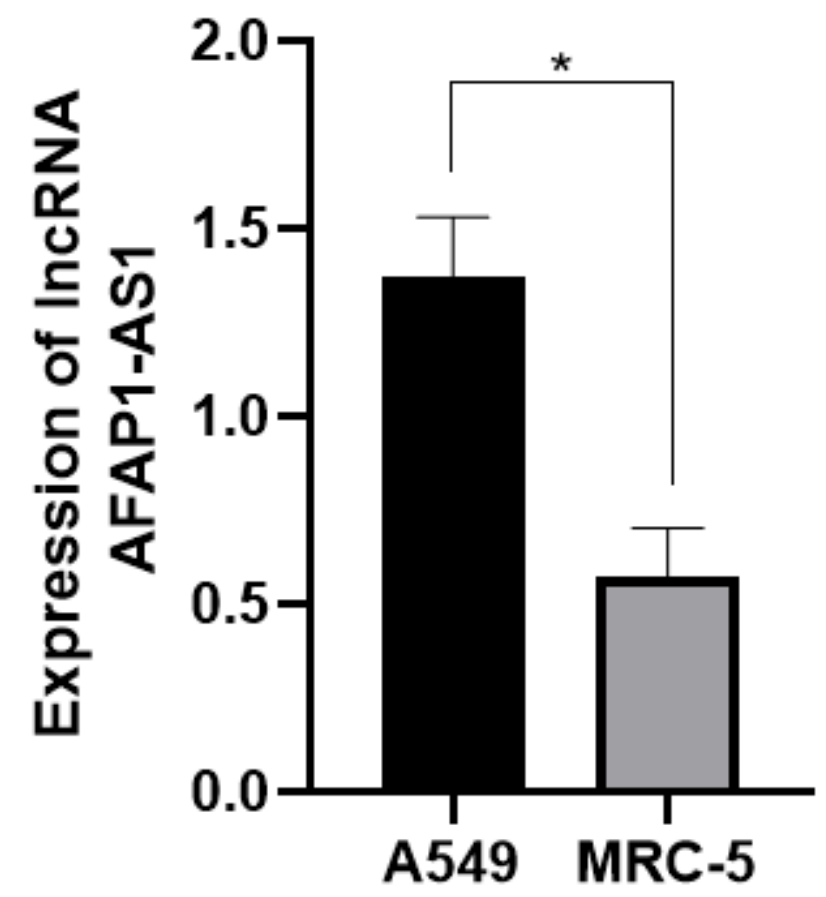

Figure 6

AFAP1-AS1 expression in NSCLS cell line (A549) and human lung epithelial cells (MRC-5). *: P $<0.05$ 


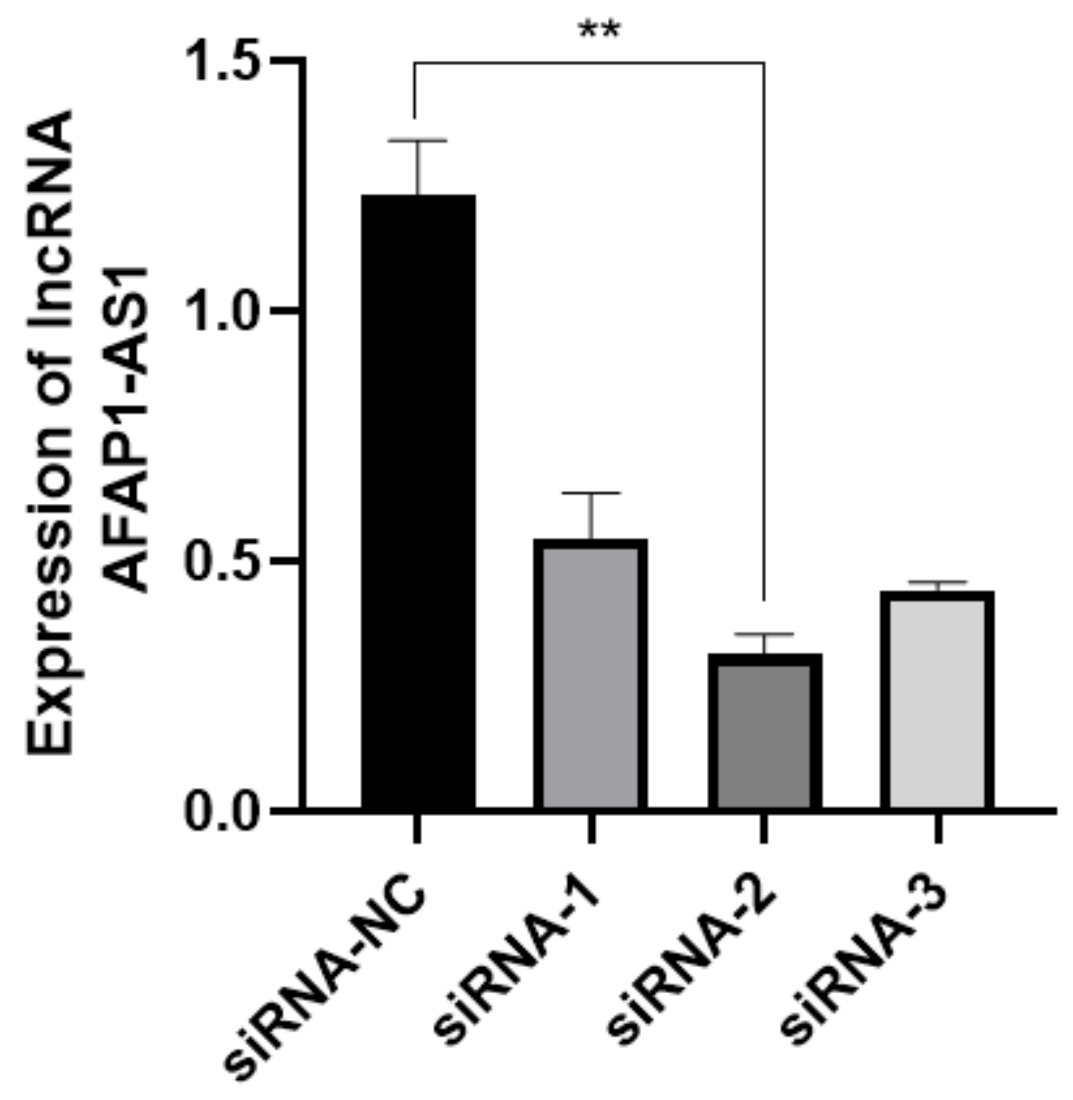

Figure 7

AFAP1-AS1 expression in A549 cells transfected with siRNA-NC, siRNA-1, siRNA-2, and siRNA-3. **: P< 0.01 . 


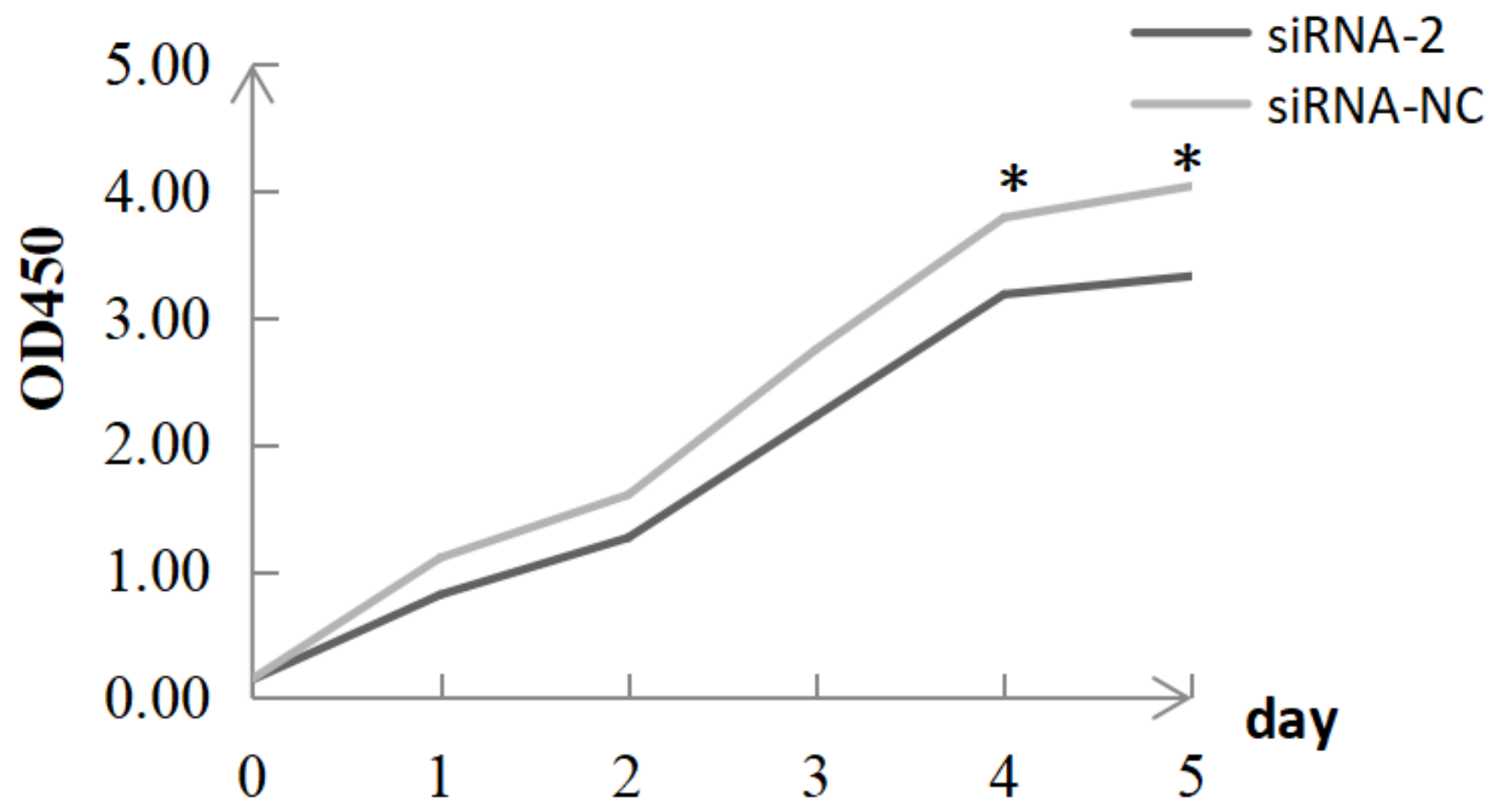

Figure 8

Cell proliferation in A549 cells transfected with siRNA-NC or siRNA-2; $*$ : $<<0.05$. 
Oh SiRNA-NC

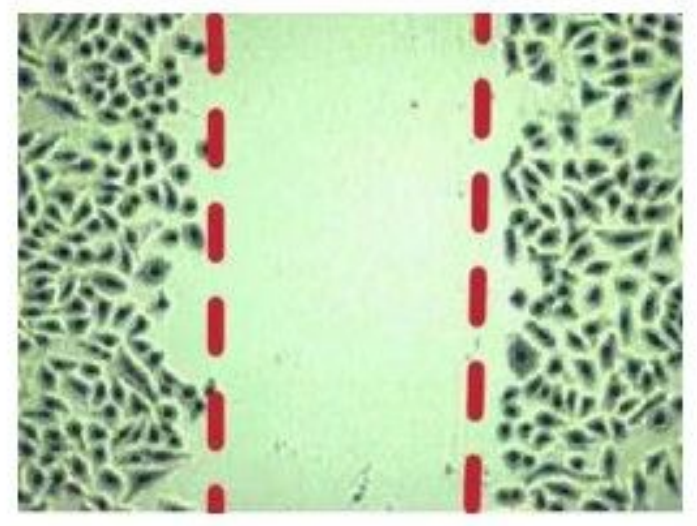

\section{8h SiRNA-NC}

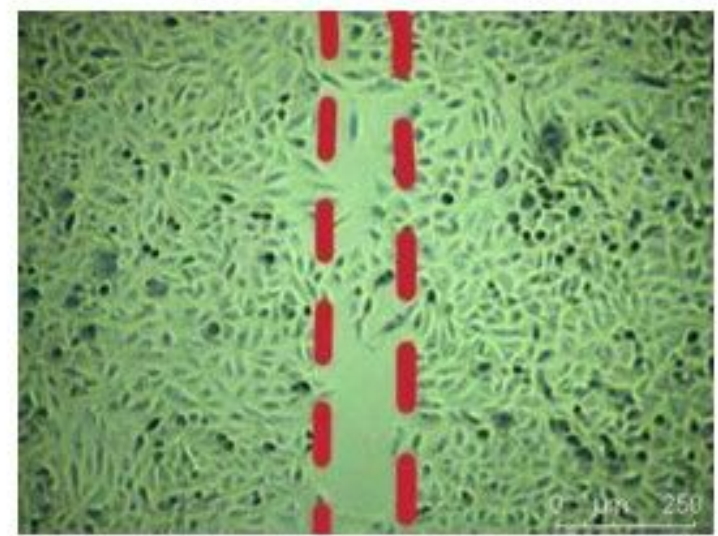

Oh siRNA-2

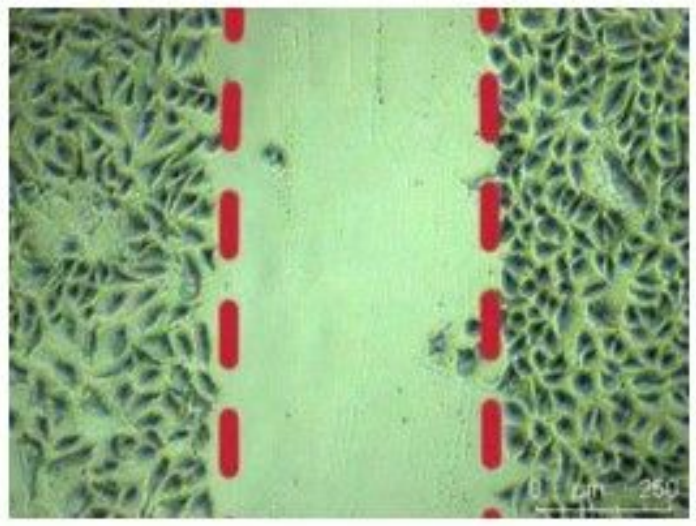

48h SiRNA-2

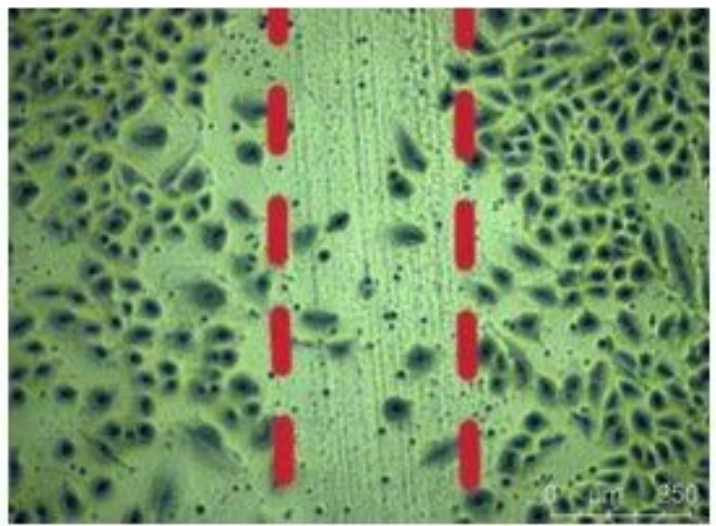

\section{Figure 9}

Evaluation of cell migratory ability in A549 cells after transfection with siRNA-NC and siRNA-2 $(P<0.05)$. 
SiRNA-2

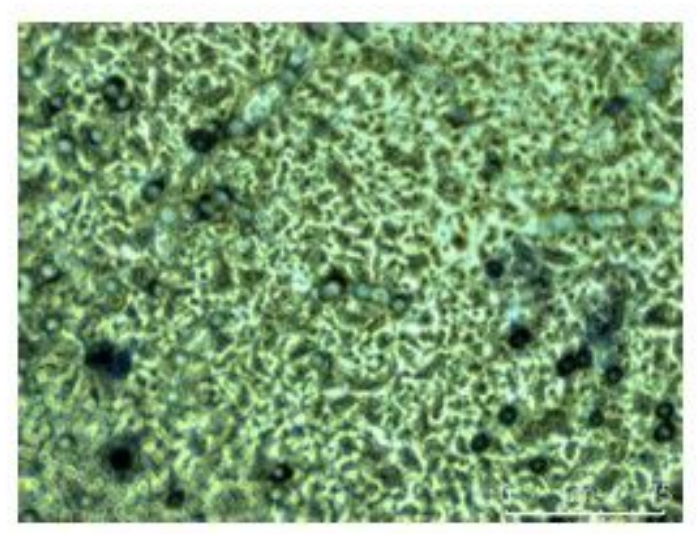

SiRNA-NC

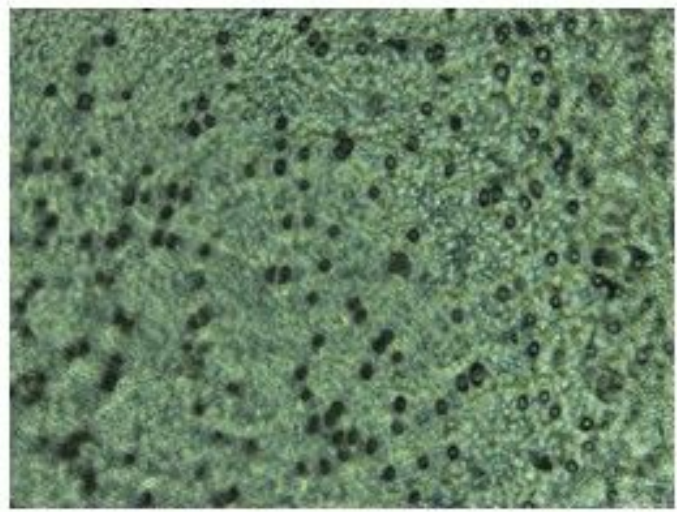

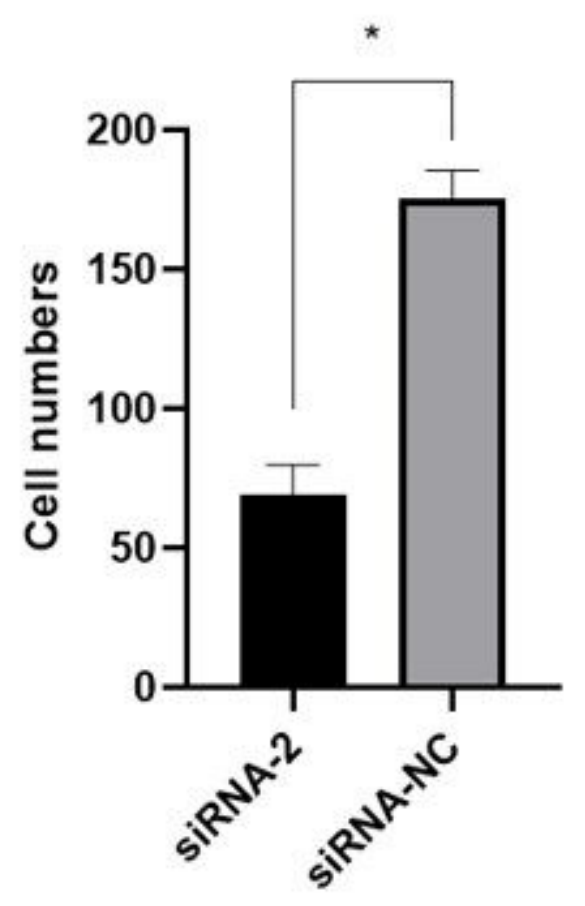

Figure 10

Cell invasive ability in A549 cells after transfection with siRNA-2 or siRNA-NC $(P<0.05)$. 

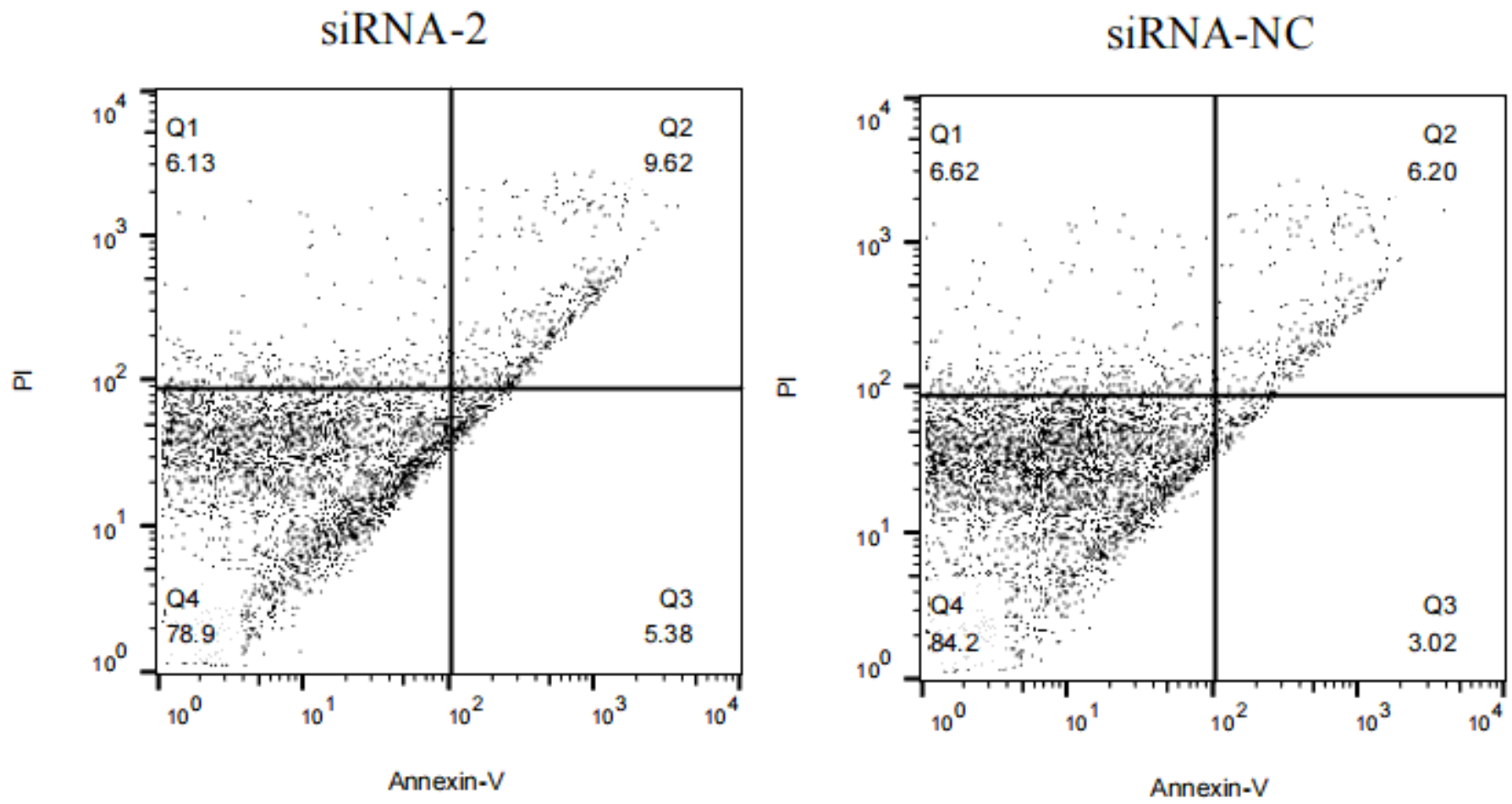

Figure 11

Cell apoptosis ability in A549 cells after transfection with siRNA-2 or siRNA-NC.

\section{Supplementary Files}

This is a list of supplementary files associated with this preprint. Click to download.

- supplementtable.doc 